\title{
SANDPILES AND SUPERCONDUCTORS: DUAL VARIATIONAL FORMULATIONS FOR CRITICAL-STATE PROBLEMS
}

\author{
J.W. Barrett ${ }^{1}$ and L. Prigozhin ${ }^{2}$ \\ ${ }^{1}$ Dept.of Mathematics, Imperial College,London SW72AZ,UK, jwb@ic:ac.uk \\ ${ }^{2}$ Blaustein Institute for Desert Research, Ben-Gurion University, Sede-Boqer Campus 84990, \\ Israel, leonid@cs.bgu.ac.il
}

\begin{abstract}
Similar evolutionary variational inequalities appear as variational formulations of continuous models for sandpile growth, magnetization of type-II superconductors, and evolution of some other dissipative systems characterized by the multiplicity of metastable states, long-range interactions, avalanches, and hysteresis. Such formulations for sandpile and superconductor models are, however, convenient for modeling only some of the variables (evolving pile shape and magnetic field for sandpile and superconductor models, respectively). The conjugate variables (the surface sand flux and the electric field) are also of interest in various applications. Here we derive dual variational formulations, similar to mixed variational inequalities in plasticity, for the sandpile and superconductor models. These formulations are used in numerical simulations and allow us to approximate simultaneously both the primary and dual variables.
\end{abstract}

keywords: variational inequalities, critical-state problems, duality, numerical solution.

\section{Introduction}

Sandpiles and type-II superconductors are examples of spatially extended open dissipative systems which have infinitely many metastable states but, driven by the external forces, tend to organize themselves into a marginally stable "critical state" and are then able to demonstrate almost instantaneous long-range interactions. The evolution of such systems is often accompanied by sudden collapses, like sandpile avalanches, and hysteresis. Although these are dissipative systems of a different nature, their continuous models are equivalent to similar variational (or quasivariational) inequalities (see [1] and the references therein). The origin of this similarity is that these models are quasistationary models of equilibrium and the multiplicity of metastable states is a consequence of a unilateral equilibrium condition. Typically, dynamics of

Please use the following format when citing this chapter:

Barrett, J.W., and Prigozhin, L., 2006, in IFIP International Federation for Information Processing, Volume 202, Systems, Control, Modeling and Optimization, eds. Ceragioli, F., Dontchev, A., Furuta, H., Marti, K., Pandolfi, L., (Boston:

Springer), pp. 25-29. 
such a system occur at the border of equilibrium and the system in a marginally stable state, often called critical. The rate with which these systems adjust themselves to the changing external conditions is determined implicitly and appears in the model as a Lagrange multiplier. Typically, the multiplier is eliminated in transition to a variational formulation written in terms of a "primary" variable (surface of a sandpile, magnetic field in a superconductor, stress tensor in elastoplasticity, etc.) In many situations, however, the Lagrange multiplier or, equivalently, a "dual" variable (sand flux upon the pile surface, electric field, and strain tensor, respectively) also has to be found. We present, for both the sandpile and the superconductivity problem, the variational formulations written for the dual variables. On discretization these dual formulations yield an efficient algorithm to compute the dual and primal variables simultaneously. Only the simplest version of each problem is considered.

\section{Sandpiles}

Let sand be poured out onto a rigid support surface, $y=h_{0}(x)$, given in a domain $\Omega \subset R^{2}$ with boundary $\partial \Omega$. If the support boundary is open, a model for pile surface evolution can be written as

$$
\partial_{t} h+\nabla \cdot \underline{q}=w,\left.\quad h\right|_{t=0}=h_{0},\left.\quad h\right|_{\partial \Omega}=\left.h_{0}\right|_{\partial \Omega},
$$

where $h$ is the pile surface, $w \geq 0$ is the given source density, $q$ is the horizontal projection of the flux of sand pouring down the pile surface. If the support has no slopes steeper than the sand angle of repose, $\left|\nabla h_{0}\right| \leq \gamma=\tan \alpha$, the simplest constitutive relations for this model read: (i) the flux $\underline{q}$ is directed towards the steepest decent of the surface, (ii) the surface slope cannot exceed the critical angle $\alpha$, and (iii) the flux is zero upon subcritical slopes. Equivalently, one can write $q=-m \nabla h$ and show that $m(x, t) \geq 0$ is the Lagrange multiplier related to the constraint $|\nabla h| \leq \gamma$. The model can be rewritten as a variational inequality for $h$,

$$
h(., t) \in K:\left(\partial_{t} h-w, \varphi-h\right) \geq 0 \forall \varphi \in K,\left.\quad h\right|_{t=0}=h_{0},
$$

where $K=\left\{\varphi \in H^{1}(\Omega):|\nabla \varphi| \leq \gamma\right.$ a.e., $\left.\left.\varphi\right|_{\partial \Omega}=\left.h_{0}\right|_{\partial \Omega}\right\}$ and $(\cdot, \cdot)$ is the standard $L^{2}(\Omega)$ inner product. Simple analytical solutions of this inequality describe piles generated on the support $h_{0} \equiv 0$. For the point source $w=\delta\left(x-x_{0}\right)$, a conical pile with critical slopes grows until its base touches the domain boundary. Then there appears a runway connecting the cone apex with the boundary and the pile growth stops: all additional sand just follows the runway and leaves the system. On the other hand, if $w>0$ everywhere in $\Omega$, the final stationary shape of the pile is different: $h(x)=\gamma \operatorname{dist}(x, \partial \Omega)$. For $w \geq 0$, the general stationary solution and an integral representation formula for the corresponding Lagrange multiplier $m$, determining the surface sand flux 
$q$, have also been obtained recently (see [2] and the references therein). Note, however, that it is not easy to compute the Lagrange multiplier using this formula. In the non-stationary case, determining the surface flux $q$ remains difficult even if the unique solution $h$ to (1) is found. To compute both these variables simultaneously, we derive a dual variational formulation of the evolutionary problem.

Let $\{h, q\}$ satisfy the model relations (i)-(iii). Then, for any test field $\psi$, $\nabla h \cdot(\underline{\psi}-\underline{q}) \geq-|\nabla h||\underline{\psi}|-\nabla h \cdot \underline{q}=-|\nabla h||\underline{\psi}|+\gamma|\underline{q}| \geq-\gamma|\underline{\psi}|+\gamma|\underline{q}|$. Hence, $(\nabla h, \bar{\psi}-\underline{q}) \geq \phi(\underline{q})-\phi(\underline{\psi})$, where $\phi(\underline{q})=\gamma \int_{\Omega}|\underline{q}|$. Since $(\nabla h, \underline{\psi}-\underline{q})=$ $\oint_{\partial \Omega} h_{0}\left\{\underline{\psi}_{n}-\underline{q}_{n}\right\}-(h, \nabla \cdot\{\underline{\psi}-\underline{q}\})$, where $\underline{\psi}_{n}$ is the normal component of $\underline{\psi}$ on $\partial \Omega$, we have $\phi(\underline{\psi})-\phi(\underline{q})-(h, \nabla \cdot\{\underline{\psi}-\underline{q}\})+\oint_{\partial \Omega} h_{0}\left\{\underline{\psi}_{n}-\underline{q}_{n}\right\} \geq 0$. Noting that $h=h_{0}+\int_{0}^{t} w d t-\nabla \cdot\left\{\int_{0}^{t} \underline{q} d t\right\}$ we finally obtain

$$
\underline{q}(., t) \in V:\left(\nabla \cdot\left\{\int_{0}^{t} \underline{q} d t\right\}, \nabla \cdot\{\underline{\psi}-\underline{q}\}\right)+\mathcal{F}(\underline{\psi}-\underline{q})+\phi(\underline{\psi})-\phi(\underline{q}) \geq 0
$$

for any $\underline{\psi} \in V$. Here $\mathcal{F}(\underline{u})=\oint_{\partial \Omega} h_{0} \underline{u}_{n}-\left(h_{0}+\int_{0}^{t} w d t, \nabla \cdot \underline{u}\right)$ and we define $V=\left\{\bar{\psi} \in[\mathcal{M}(\Omega)]^{2}: \nabla \cdot \psi \in L^{2}(\Omega)\right\}$, where $\mathcal{M}(\Omega)$ is the Banach space of bounded Radon measures. Existence of a solution to problem (2) is proved in [3].

To approximate (2) numerically, we smoothed the non-differentiable functional $\phi$ by introducing $|\underline{q}|_{\varepsilon}=\left(|\underline{q}|^{2}+\varepsilon^{2}\right)^{1 / 2}$, discretized the regularized equality problem in time, employed Raviart-Thomas finite elements of lowest order with vertex sampling on the nonlinear term, and solved the resulting nonlinear algebraic system at each time level iteratively using a form of successive overrelaxation (see Fig. 1 for an example of a numerical simulation).
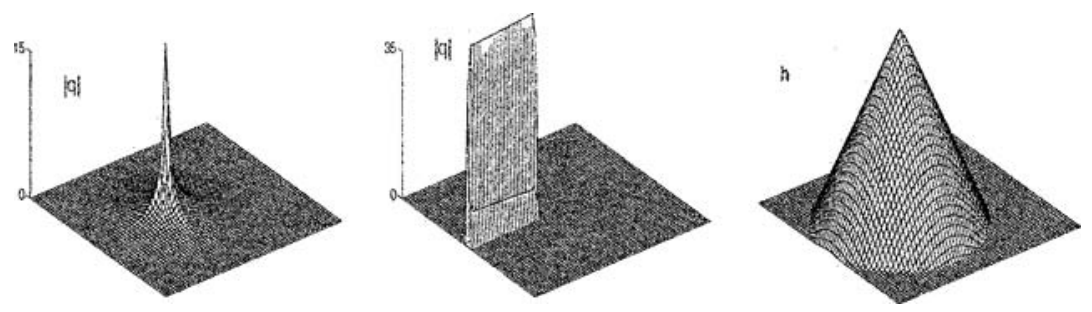

Figure 1. Point source above square support with $h_{0}=0$. The cone grows until its base touches the support boundary and the runway appears. Shown: 1) sand flux $|\underline{q}|$ before and after this moment; 2) the final pile shape computed as $h=h_{0}+\int_{0}^{t} w d t-\nabla \cdot\left\{\int_{0}^{t} \underline{q} d t\right\}$. 


\section{Superconductors}

Phenomenologically, the magnetic field penetration into type-II superconductors can be understood as a nonlinear eddy current problem. Let a long cylindrical superconductor with a simply connected cross-section $\Omega$ be placed into a non-stationary uniform external magnetic field $\underline{h}_{e}(t)$ parallel to the cylindrical generators. According to Faraday's law, time variations of this field induce in a conductor an electric field $\underline{e}$ leading to a current $j$ parallel to the cross-section plane; this current induces a magnetic field $\underline{h}(x, t)$ parallel to $\underline{h}_{e}$. Omitting the displacement current in Maxwell's equations and scaling the magnetic permeability to be unity, we obtain the following model,

$$
\partial_{t}\left(h+h_{e}\right)+\operatorname{curl} \underline{e}=0, \operatorname{curl} h=\underline{j},\left.\quad h\right|_{t=0}=h_{0}(x),\left.\quad h\right|_{\partial \Omega}=0,
$$

where curl $\underline{u}=\partial_{x_{1}} u_{2}-\partial_{x_{2}} u_{1}$ and $\operatorname{curl} \underline{u}=\left(\partial_{x_{2}} u,-\partial_{x_{1}} u\right)$. Instead of the usual Ohm law, a multivalued current-voltage relation (the Bean model) is often employed for type-II superconductors. It is postulated that (i) the electric field $\underline{e}$ and the current density $j$ have the same direction, (ii) the current density cannot exceed some critical value, $j_{c}$, and (iii) if the current is subcritical, the electric field is zero. One can write $\underline{e}=\rho \underline{j}$ and show that the effective resistivity, $\rho(x, t) \geq 0$, is the Lagrange multiplier related to current density constraint $|\underline{j}| \leq j_{c}$. Using conditions (i)-(iii) we can eliminate the electric field from the model. This yields the variational inequality,

$$
h(., t) \in K:\left(\partial_{t}\left\{h+h_{e}\right\}, \varphi-h\right) \geq 0 \forall \varphi \in K,\left.h\right|_{t=0}=h_{0},
$$

where $K=\left\{\varphi \in H_{0}^{1}(\Omega):|\nabla \varphi| \leq \gamma\right.$ a.e. $\}$. This inequality for $h$ can be approximated numerically and often even solved analytically. However, computing the electric field $\underline{e}$ may be difficult [4]. As for the sandpile model, a dual variational formulation can be derived to find both variables simultaneously:

$\underline{e}(., t) \in W:\left(\operatorname{curl}\left\{\int_{0}^{t} \underline{e} d t\right\}, \operatorname{curl}\{\underline{\psi}-\underline{e}\}\right)+\mathcal{F}(\underline{\psi}-\underline{e})+\phi(\underline{\psi})-\phi(\underline{e}) \geq 0$

for any $\psi \in W$, where $W=\left\{\psi \in[\mathcal{M}(\Omega)]^{2}: \operatorname{curl} \psi \in L^{2}(\Omega)\right\}, \phi(\underline{u})=$ $\int_{\Omega} j_{c}|\underline{u}|$, and $\mathcal{F}(\underline{u})=\left(h_{e}(t)-\overline{h_{e}}(0)-h_{0}\right.$, curl $\left.\underline{u}\right)$. The primary variable, $h$, is found as

$$
h=h_{0}+h_{e}(0)-h_{e}(t)-\operatorname{curl}\left\{\int_{0}^{t} \underline{e} d t\right\} .
$$

The simple transformation $R: \underline{e}=\left(e_{1}, e_{2}\right) \rightarrow\left(e_{2},-e_{1}\right)$ maps $W$ to $V$ and enables us to use the same Raviart-Thomas finite element as in the previous case. To model the magnetization of a superconductor with a multiply connected cross-section (Fig. 2) we "filled" the hole and set $j_{c}=0$ there.

\section{References}

[1] L. Prigozhin. Variational inequalities in critical-state problems. Physica D 167:197-210, 2004. 

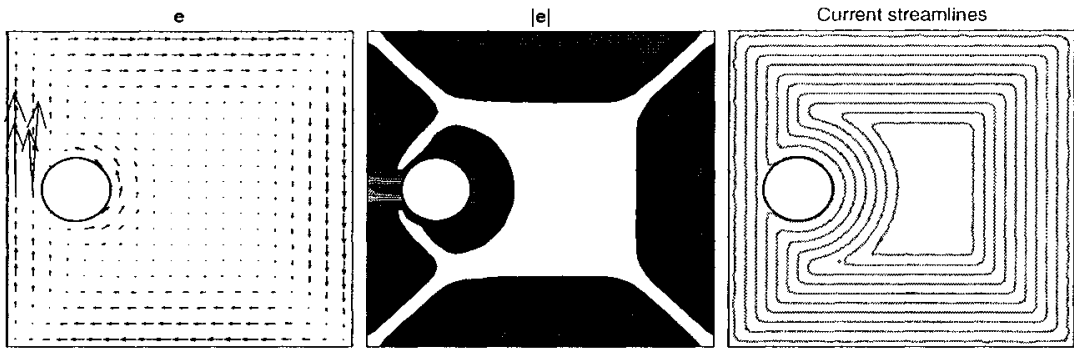

Figure 2. Cylindrical superconductor in a growing field (square cross-section with a hole, zero initial state). Shown at the same moment in time: the electric field e, level contours of $|\underline{e}|$, and the current streamlines plotted as levels of $h$. Note the "runway" (red region in the $|\underline{e}|$ contour plot) through which the magnetic field penetrates the hole and where the electric field is the strongest.

[2] P. Cannarsa, P. Cardaliaguet. Representation of equilibrium solutions to the table problem for growing sandpiles. J. Eur. Math. Soc. 6:435-464, 2004.

[3] J.W. Barrett, L. Prigozhin. Dual formulations in critical-state problems. In preparation.

[4] A. Badía-Majós, C. López. Electric field in hard superconductors with arbitrary cross section and general critical current law. J. Appl. Phys. 95:8035-8040, 2004. 